\title{
How to get the Best Results in Transaxillary Breast Augmentation with Anatomic form Stable Implants: Pocket Shapes, Insertion, and Maintenance
}

\author{
Jin Seok Park ${ }^{1}$, Joong Hyuk Yim², Jae Jin Ock ${ }^{3}$ Sang Hoon Sun ${ }^{4}$, \\ Young Woo Lee ${ }^{5}$, Sung Chul Park ${ }^{6}$, Chul Hwan Seul ${ }^{7}$, Won Joune Yoon ${ }^{8}$
}

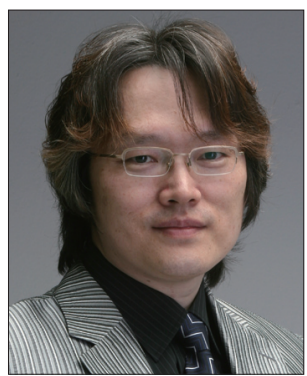

${ }^{1}$ Parkjinseok Aesthetic Clinic, Seoul; ${ }^{2}$ TL Plastic Surgery Clinic, Seoul; ${ }^{3}$ THE Plastic Surgery Clinic, Seoul; ${ }^{4}$ BR Plastic Surgery Clinic, Seoul; ${ }^{5}$ Leeview Plastic Surgery Clinic, Seongnam; ' $U B A$ Plastic Surgery Clinic, Seoul; ${ }^{7} J W$ Plastic Surgery Clinic, Seoul; ${ }^{8}$ Migo Aesthetic Surgical Clinic, Seoul, Korea

Although IMF incision is known the best way for anatomic implant, most Korean doctors and patients hesitate IMF incision. Anatomic form stable implants have some benefits such as less prominent upper pole, less wrinkles and ripples, and less rupture rate than round cohesive type I implants. However more concern is necessory for placing the anatomic implants. The Korean Academic Association of Breast Surgery(KAABS) planned to support some tips for using anatomic form stable implants through axillary incision. The KAABS gathered and analyzed the concepts of Korean plastic surgeons who have experienced transaxillary breast augmentation with anatomic form stable implants. The KAABS requested them of their concepts of 9 basic categories: entrance dissection, pocket dissection, lubricant, inserting aids, skin protector, inserting direction, suction drainage, dressing, compression garment, and their key considerations. Eight expert surgeons suggested their own cutting edge methods of transaxillary breast augmentation with the anatomic form stable implant, however each surgeon should find his or her own method. Authors and KAABS hope that these developing and incomplete concepts help beginners to find their own concepts.

(Arch Aesthetic Plast Surg 19: 106, 2013)

Key word: Breast implants, Anatomic model, Axilla incision, Augmentation mammaplasty

\section{INTRODUCTION}

Anatomic form stable implants were introduced in Europe in 1993. In Korea, we can use these implants from 2012. As new generation anatomical implants are highly cohesive and tear-drop shape, long incision and short distance to the final destination is desirable to insert them easily and prevent rotation. ${ }^{1}$ These implants have some benefits such as less prominent upper pole, less wrinkles and ripples, and less rupture rate than

Received April 5, 2013

Revised June 11, 2013

Accepted June 12, 2013

Address Correspondence: Jin Seok Park, M.D., Ph.D., Parkjinseok Aesthetic Clinic, B 111, JB Miso Bd. 841 Nonhyeon-ro, Gangnamgu, Seoul 135-892, Korea. Tel:+82-2-514-0500, Fax:+82-2-511-0400, Email: breastpark@naver.com

${ }^{*}$ This report is based on a Symposium of Korean Academic Association of Breast Surgery (KAABS) which was held on March 9 of 2013. round cohesive type I implants. ${ }^{1-4}$ This anatomic form stable implants were approved by Korean FDA in 2012. Although axillary approach is the most popular in Korea, there is no paper for inserting anatomic implants through axillary incision among many previous reports. So for reducing trial and errors of transaxillary breast augmentation with anatomic form stable implant, The Korean Academic Association of Breast Surgery (KAABS) gathered and analyzed the concepts of Korean plastic surgeons who have experienced transaxillary breast augmentation with anatomic form stable implants.

Contrary to round implant the axis of the implant should be maintained accurately in the pocket and the textured surface hinders easy manipulation of the implant in the pocket, care should be taken from the insertion of the implant.

\section{MATERIAL AND METHODS}

The KAABS gathered and analyzed the considerations for 
the best results of transaxillary breast augmentation with anatomic form stable implants as 8 basic categories of operation procedures; Entrance dissection, Pocket dissection, Lubricant, Inserting aids such as Sleeves, Keller funnel, etc Skin protector, Inserting direction, Drains as Hemovac, Dressing and Garment. And 8 speakers talked about their key concepts for transaxillary breast augmentation with anatomic form stable implants.

The KAABS requested answers of 8 basic categories as below to 8 speakers.

1. Entrance dissection for conduit

E1: wide subcutaneous pocket + narrow intermuscular space

E2: wide subcutaneous pocket + wide intermuscular space

E3: no extra subcutaneous pocket +wide intermuscular space

E4: other concepts

2. Pocket dissection for implants

$\mathrm{P} 1$ : narrow upper pole + wide lower pole as implant for fitting

P2: wide upper pole for easily inserting and managing implant + wide lower pole as implant for fitting

P3: other concepts

3. Lubricant

L1: Lidocaine jelly

L2: Antibiotic ointment

L3: Others

L4: no lubricant

4. Inserting aids

A1: no

A2: pastrybag

A3: Keller funnel

5. Skin protector

S1: Tegaderm ${ }^{\circledR}$

S2: others as Duoderm

S3: no

6. Inserting direction: inserting

D1: larger lower pole first + turning implant as 90 degree (rotating outer to lower)

D2: larger lower pole first without turning implant

D3: smaller upper pole first + turning implant after inserting

D4: smaller upper pole first without turning implant

D5: other concepts

\section{Hemovac}

H1: Hemovac for collapse pocket + drainage (maintaining hemovac without drainage)

$\mathrm{H} 2$ : Hemovac for drainage
H3: no Hemovac

8. Dressing and Garment

B: duration of bandage-no bandage: B0, bandage for 7 days: B7

T: duration of taping-no taping: T0, taping for 7 days: T7

G: duration of Garment or elastic band-garment for 8 weeks: G8

\section{RESULTS}

1. Answers of 8 basic categories of operation procedures are as Table I.

2. Key considerations for the best results of each speakers are as belows.

\section{A. Jinseok Park's key concept (Fig. 1)}

Is reducing possibilities of double capsule phenomenon. For this he makes the fitted pocket for the shaped implant as wide lower pole and narrow upper pole and maintaining narrow conduit. He makes dual plane and wider lower lateral area for reducing tension of the lower pole. For the narrow conduit, Jinseok Park adopts 4 concepts; (1) making wide subcutanous dissection from the incision site, (2) division of thracic fascia(deep layer of superficial fascia) for reducing the tension of the lateral soft tissue, (3) making subfascial pocket around entrance for easy expansion, (4) using lubricant(sterilized lidocaine jelly). Jinseok Park uses a kind of pastry bag for easy insertion and maintaining direction. Jinseok Park fixes implants with Fixo$\mathrm{mul}^{\circledR}$ and elastic bandage for 3 days. Jinseok Park restricts heavy exercise of shoulder joint for 8 weeks except stretching and apply elastic band on the upper pole of breasts for 8 weeks.

\section{B. Joonghyuk Yim's key concept (Fig. 2)}

Before inserting the implant, subcutaneous tunneling has to be done widely with thoracoepigastric vessels ligated or cauterized. Also pocket has to be made a little bit larger than the implant to adjust the implant when there is little deviation of the implant. When inserting the implant, upper pole of the implant is directed downward. In case of right breast, left hand is gently grasping the implant and the second finger of right hand is used to push and rotate the implant simultaneously inside the subcutaneous and subpectoral space. I call it "pivotal rotation" insertion. After implant is inserted into the subpectoral space just beyond the skin incision, the implant is almost 120 $\sim 150$ degrees rotated. With final push with the second finger the implant comes down into the pocket with residual rotation to 180 degrees. Since subpectoral tunnel was widely dissected 
Table I. Brief summary of Results

\begin{tabular}{lcccc} 
& Entrance & Pocket & Lubricant & Sleeves \\
\hline JS Park & E1 & P1 & Lidocaine jelly & pastry bag \\
JH Yim & E2 & larger than implant & Betadine & no \\
\hline J Ock & sufficient space & comfortable & Lidocaine jelly & no \\
SH Sun & E2 & P1 & General jelly & no \\
YW Lee & E2 & P2 & Lidocaine jelly & no \\
SC Park & E2 & P1 & Antibiotic oint & no \\
CH Seul & E3 & P1 & Betadine & no \\
WJ Youn & E2 & P2 & Antibiotic oint & no \\
\hline
\end{tabular}

1. Entrance dissection for conduit. E1: wide subcutaneous pocket+narrow intermuscular space E2: wide subcutaneous pocket+wide intermuscular space E3: no extra subcutaneous pocket +wide intermuscular space $\mathbf{E} 4$ : other concepts

2. Pocket dissection for implants. P1: narrow upper pole+wide lower pole as implant for fitting P2: wide upper pole for easily inserting and managing implant+wide lower pole as implant for fitting P3: other concepts

3. Lubricant. L1: Lidocaine jelly L2: Antibiotic ointment L3: Others L4: no lubricant

4. Inserting aids. A1: no A2: pastry bag A3:Keller funnel

\begin{tabular}{lllll}
\hline & Skin protector & Direction & Hemovac & Dressing \\
\hline JS Park & no & D2 & no & B3 T3 G8 \\
JH Yim & no & D3 & H2 & no \\
\hline J Ock & no & D2, D4 68 \\
\hline SH Sun & Tegaderm & Side first & no & B5 G4 \\
\hline YW Lee & Duoderm & D3 & H2 & T5 G7 \\
SC Park & Tegaderm & D1 & H1 & T5 G8 \\
\hline CH Seul & no & Side first & H2 & T21 G10 \\
WJ Youn & no & Counterclockwise $150^{\circ}$ & no & B3 T7 G5 \\
\hline
\end{tabular}

5. Skin protector. S1: Tegaderm ${ }^{\circledR}$ S2: others as Duoderm ${ }^{\circledR}$ S3: no

6. Inserting direction: inserting. D1: larger lower pole first+turning implant as 90 degree(rotating outer to lower) D2: larger lower pole first without turning implant D3: smaller upper pole first+turning implant after inserting D4: smaller upper pole first without turning implant D5: other concepts

7. Hemovac H1: Hemovac for collapse pocket+drainage (maintaining hemovac without drainage) H2: Hemovac for drainage H3: no Hemovac

8. Dressing and Garment. B: duration of bandage-no bandage: B0, bandage for 7days:B7 T: duration of taping - no taping:T0, taping for 7days:T7 G: duration of Garment or elastic band - garment for 8 weeks: G8

\section{(A)}

Fig. 1. Jinseok Park's Key concept (A) Narrow passage from axillary incision for the Anatomic form stable implant. (1) Blue area: subcutaneous dissection, (2) White line: cutting line of thoracic fascia, (3) Orange area: subfascial dissection for enhancing flexibility, (4) Yellow area: passage under the pectoralis major, (5) Purple line: meridian line. (B) Using lubricant and a kind of pastry bag is beneficial for easy inserting the implants without injury to the implant or skin. 
compression in this area is essential. Hemovac is maintained $2 \sim 4$ days which is a little longer than round implant cases. And all the compressvie dressings and garment is recommended thoroughly than the round implant cases also.

\section{Jaejin Ock's key concept (Fig. 3)}

Key concept is comportability for control to implants and balanced tension for preventing from rotation. Incision length is 4 to $4.5 \mathrm{~cm}$ and subcutaneous pocket and opening the pectoral fascia is sufficient enough to insert implants without gel fracture. Implants pocket should be made to have a comportable space to reduce the tension and induce the tissue redrapping. Implant position without excessive tension would be maintained in spite of wound contraction, change in posture. But the change in position should be possible by hands. Always I make a dual plane pocket. I try to make a balanced tension in all directions without bias. I insert the implants from the narrow part depending on the shape and rotated at the same time if it needs. After meticulous hemostasis, hemovac is not needed usually. Elastic bandage is maintained for 4 to 5 days, garment is applied for 4 weeks for the purpose of maintaining the position of implants.

\section{Sanghoon Sun's key concept (Fig. 4)}

About $4 \mathrm{~cm}$ length skin marking in the deepest part of the
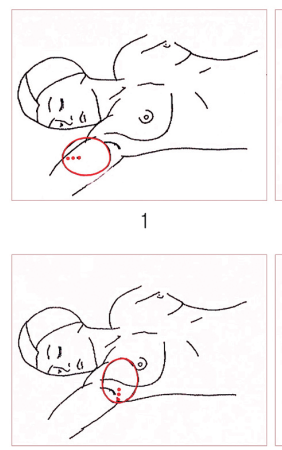

4

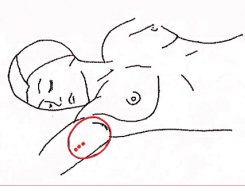

2

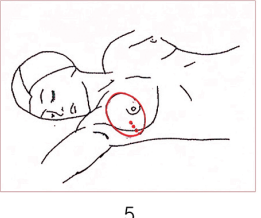

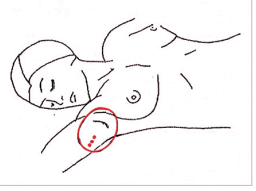

3

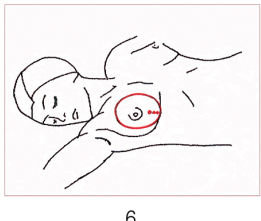

6
Fig. 2. Joonghyuk Yim's pivotal rotation.

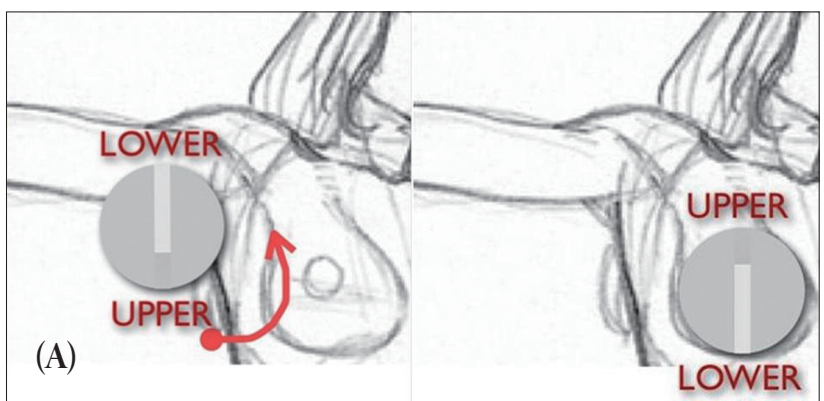

armpit to avoid the visible incisional scars is designed as dissection location. The skin is incised and subcutaneous dissection is carried out $3 \mathrm{~cm}$ toward the border of the pectoralis major muscle. Open the layer between the pectoralis major and minor muscle, and make the space with spreader equipment for the window to allow access of endoscope. The direction of the pocket dissection is conducted from the superomedial, down to inferomedial, inferolateral upto superolateral pocket in a clockwise fashion on right breast. After the dissection of pocket, complete the bleeding control and irrigate the pocket with solution. Before the insertion of implant, take on the new gloves, and implant is immerged in betadine-saline solution. For the skin protection, attach the Tegaderm ${ }^{\circledR}$ on armpit. Paste general jelly without lidocaine at the side edge of implant, after that insert the implant with turning manner as 180 degree rotation. Do not use the reduced height style 410 implant, because it is oval shaped that raise the possibility of rotation and relatively firm. Insertion of large implant more than $350 \mathrm{~g}$ is more difficult due to firmness. So, Replicon'or full height style 410 implant would be preferable. After operation, apply the elastic tapes around the breast mount during 5 days, and garment with ace band during 1 week for preventing of rotation.

\section{E. Youngwoo Lee's key concept (Fig. 5)}

For Axillary incision marking, $4 \mathrm{~cm}$ of designing can be done at about $1 \mathrm{~cm}$ apart from the rear p. major lateral border. And

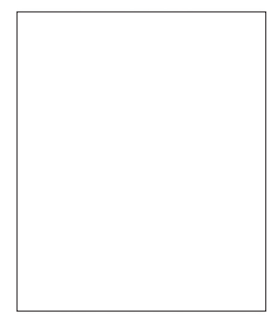

Polytech

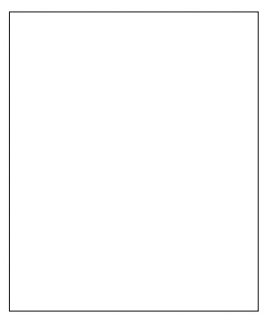

Allergan Full Height

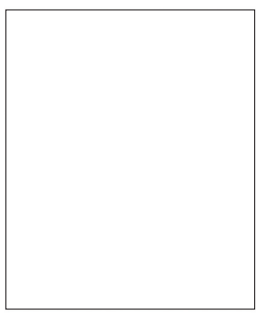

Allergan Reduced Height
Fig 3. Jaejin Ock’s key concept.

Fig. 4. Sanghoon Sun's Key concept (A) Insertion of implant: Side first + turning implant as 180 degree rotation. (B) Fixation with taping for 5 days, and garment with ace band for 1 week. 
tattooing can be made at the center of incision for accurate approximation.

When conducting dissection, it is necessary to preserve the axillary pocket pad in the inferior of incision to prevent depressed scar (tissue); and protect the margin with a plastic cap to avoid skin maceration. I used an endoscope, an electrocautery dissector, triangular shape and mini-spatular shape blunt dissectors which are commonly used.

When the subpectoral plane dissection, coagulation and dissection has been done using electrocautery to prevent blood staining through the loose tissue layer in between pectoralis major muscle and minor muscle. Then, cut the origin part of pectoralis major muscle at the lower breast area. For the lateral portion, likewise, check the intercostal nerves and sweep it carefully using mini-spatular dissector.

In order to form a new IMF line, lowering the previous IMF line often occurs. In this case, confirmation of the abdominal muscle belly in the lower-lateral area of pectoralis major muscle is needed to prevent the double bubble.

Implant insertion is accessed from the lateral side keeping the surgical instruments with 45 degrees through axillar, based on the central marking band. Then starting from the upper pole, the implant rotates 90 degrees toward medial.

By checking the monitor, we can determine if the implant is well aligned to the desired base line using endoscope.

Patients will wear a postural bra for about 2 months after the operation.

It helps to prevent mal-position and to motivate a more natural fullness in the lower pole.

The patients wear wireless bra for about 6 months
We used Hemovac in every case and removed when it's less than $1 \mathrm{cc}$ per hour in 1 day of pod.

\section{F. Sungchul Park's key concept (Fig. 6)}

The key concept of my surgery is to dissect the upper breast widely, to help aligning the implant with the breast meridian as the implant working place, and then dissect of lower breast, resulting in tight fit to implant.

After the skin is incised, dissect subcutaneous tissue widely, incise lateral pectoral fascia long and make a wider subpectoral tunnel so that the implant can be easily inserted.

According to its height, the implant is inserted differently.

For the full height implant, its larger lower pole is inserted first, and then rotated 90 degrees. It is checked if the implant' $s$ orientation is aligned with the breast meridian. Then, the implant is glided down carefully.

For the medium height implant, as it is placed with its smaller upper pole upward. Its side is inserted first. It is checked if the implant's orientation is aligned with the breast meridian. Then, the implant is glided down carefully.

To collapse the upper pole after surgery, the Hemovac is used for one or two days. Up to three weeks after surgery, the patient wear specialized bras or has the pressure bandage on the upper pole.

\section{G. Chulhwan Seul's key concept (Figs. 7, 8)}

The length of skin incision is $3.5 \sim 4 \mathrm{~cm}$ and it is $5 \mathrm{~mm}$ longer than the incision for round implant. After subcutaneous dissection to the lateral border of pectoralis major, thoracic fascia is opened widely to insert implant easily. Pocket dissection is
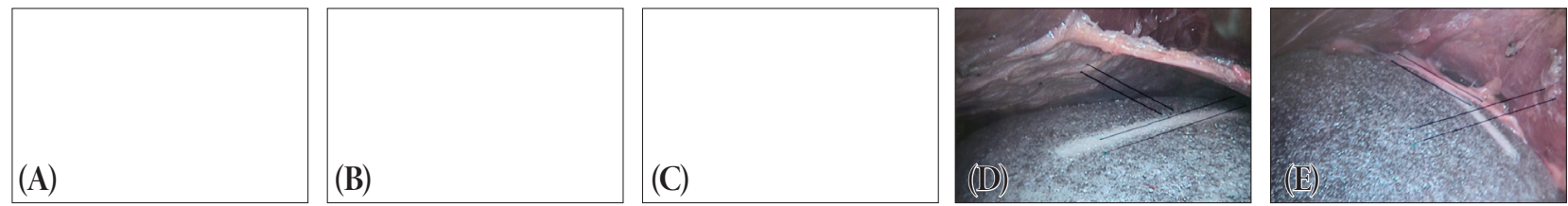

Fig. 5. (A), (B), (C): Young Woo Lee's key concept for implant insertion (right hand dominant surgeon), (D): Marking band axis check through the monitor (right side), (E): Marking band axis check through the monitor (left side).

(A)

Fig. 6. Sungchul Park's key concept. (A) Insertion of the full height implant: larger lower pole first, rotate 90 degree and glide down, (B) Insertion of medium height implant: Its side inserted first and glide down, (C) Pressure bandage on the upper pole for 3 weeks. 
performed with endoscope in the plane of dual plane type 1. The pocket should not be too wide to reduce the possibility of implant rotation and double capsule formation, but it should not be too narrow because narrow pocket can cause folding of implant edge especially in soft gel anatomic implant. Betadine solution is used as lubricant. Because Betadine itself is a disinfectant, it can reduce the possibility of bacterial contamination of implant from the skin. Usually, the implant is rotated about $180^{\circ}$ during insertion with right second finger, therefore, the implant is derotated as $180^{\circ}$ before insertion and the side part is inserted first. When I use Keller Funnel ${ }^{\mathrm{TM}}$, saline is used as lubricant and implant is not derotated before insertion.

In natural breast, lower lateral volume is larger than superomedial volume. Therefore, I don't put the anatomic implant in a vertical axis but put the axis rotated medially along the meridian line of breast in a angle of about $10^{\circ}$. This concept is beneficial even after malrotation is occurred. Most of the malrotation

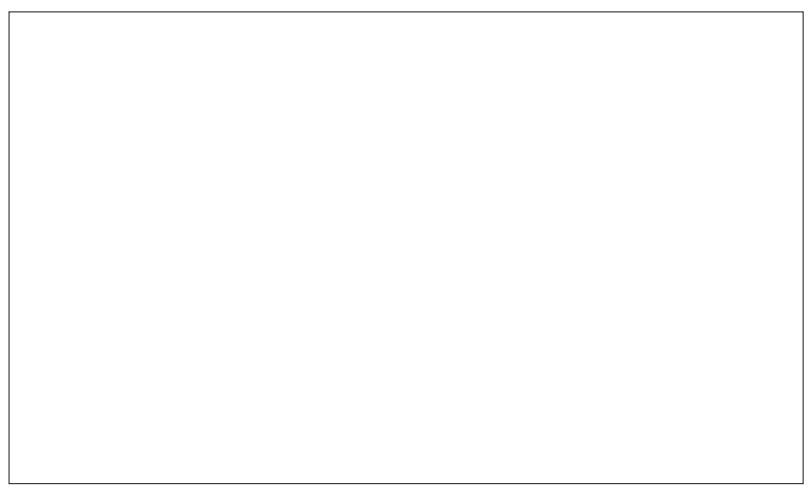

Fig. 7. Chulhwan Seul's key concept: Implant axis.

(A)

(B)

H. Wonjune Yoon's key concept (Fig. 9)

The preoperative design on the patient's chest is same as the design for IMF approach. The axillary incision is $4 \sim 5 \mathrm{~cm}$. Upper pole dissection is more wide for implant pathway and lower pole dissection is just fit to implant lower margin.

Teramycin eye ointment and low molecular Hyaluronic acid are used to impant pathway for lubrication.

For insertion of the implant, left hand grasp the implant and right 2nd finger push right margin of the implant, then the implant rotate counterclockwise and insert through the pathway. After the implant is inserted, I do let a appropriate position of implant. And then, the implant position checking is performed by touching the position maker on the implant base.

The post-operative care is almost same as the case of round type breast impant. Elastic taping is applied on upper pole during 4 days. Elastic bandage is applied on upper pole during 6 weeks, not too tight.

Fig. 8. Elastic strap: (A) V shape; desirable, (B) inverted V shape; undesirable.
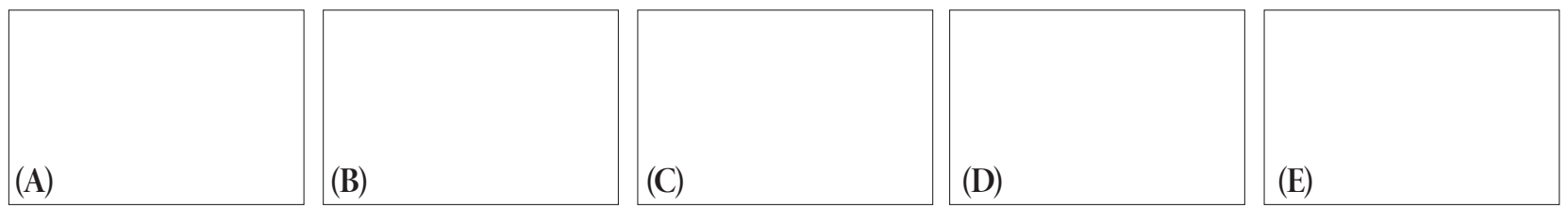

Fig. 9. Wonjune Yoon's key concept. (A) (C) implant insertion: 150 degree couterclockwise rotation, (D) implant axis control: I find the point on the skin that is projection of impalnt orientation mark, then this point is mark on the skin with GV or marking pen, (E) elastic taping is applied, especially upper pole, during post operative 4 days. 


\section{DISCUSSION}

In the Symposium there were some comments of qualified plastic surgeons other than speakers as belows.

Sang Tae Ahn, M.D. (Professor, Department of Plastic Surgery, The Catholic University, College of Medicine, Seoul, Korea):

As new generation anatomical implants are highly cohesive and tear-drop shape, long incision and short distance to the final destination is desirable to insert them easily and prevent rotation. Transaxillary approach, compared to the inframammary approach, the implants should be moved longer distance through a narrow tunnel. It needs special skill and expertise to ensure proper placement of implant and prevent tissue injury of the site of entrance and prevent deformation of the implant due to the forceful insertion through a short incision.

It may not be difficult for those experts who are doing more than hundreds of cases in a year. However, most of plastic surgeons are doing less than 10 20 breast augmentation surgeries in a year. There must be a long learning curve for those ordinary plastic surgeons to insert anatomical implant using transaxillary approach.

I recommend that the manuscript include authors' comments on their experiences about learning curve and gel fracture. How long did it take for them to use this technique comfortably and how long will it be for the ordinary plastic surgeons? Was there any chance to remove the implant immediate or late postoperatively and to find gel fracture? What do the authors think about using inframammary approach instead of transaxillary approach for those ordinary plastic surgeons to insert anatomical implant?

Soon Chan Um, M.D. (Director, Um nagumo Aesthetic Plastic Surgical Clinic, Seoul, Korea):

In the transaxillary approach, over dissection in upper lateral pocket seems to be unavoidable. For reducing the possibility of the rotation of anatomical implant, it's important to make a symmetrical pocket for implant at the least in the lower portion of the pocket and also the dissection of the upper medial portion in the pocket should be restricted.

In order to get the lower pocket symmetrical, first, a meridian line needs to be marked on the skin, starting from the mid point of upper pocket border line, passing through the mid point of the nipple or near by nipple, in cases the location of nipple is laterally one sided, and reaching the mid point of new IMF line. The next step is to design a symmetrical arch that centers the junction point where the meridian and the IMF meet, basically, it is to make one half of the arch of the new IMF (starting from sternal junction with IMF to junctional point of meridian line with new IMF) symmetrical with the other half (from junctional point of meridian line with new IMF to end point joined with ant. axillary line).

After inserting implant, axis of implant should be adjusted to the meridian line on the skin using index finger under the endoscopic view, while pricking skin with a forceps.

Hyung Bo Sim, M.D. (Director, BR Plastic Surgery Clinic, Seoul, Korea):

For using anatomical implant through the axilla, I experienced several tips. First, the reduced height implants are not recommendable through axilla. Second, the upper medial dissection in the pocket should be restricted. Third, the pocket should be made as a dual plane for balanced adjustment between pocket and implant. ${ }^{5}$ Fourth, the Pectoralis major muscle in lower sternocostal junction might often be divided partially not entirely.

Kyu Ho Cha, M.D.(Director, Logos Plastic Surgery Clinic, Daegu, Korea):

In the lower portion of breast, there is a volume difference between the lateral pole and the medial pole. The lateral portion has more volume than the medial portion does. Consequently, when using a form-stable implant, the implant needs to be orientated toward more lateral position in the lower pole of breast.

In case of a patient who has chest wall deformities such as anterior projection or depression, I prefer using standard gel implant to form-stable device because the former easily blends into the chest wall defect whereas the latter may cause implant rotation and malposition, and moreover it tends to accentuate the deformities.

Paik Kwon Lee, M.D. (Director, Apgugeong Avenue Plastic Surgery Clinic, Seoul, Korea):

In terms of incision location, I think that inframammary incision is the best for anatomic form stable implant. However the operation scar is the important consideration in oriental woman whose scar tends to be hypertrophic and takes long time, more than 1 year, to be stable even in the case of fine scar, Therefore transaxillary incision is the another option for anatomic form stable implant in terms of scar location. But transaxillary incision have some drawbacks in placing implant (to prevent malrotation) and making just fitting pocket (to prevent malposition and seroma formation) for anatomic form stable implant. So it should be careful to avoid such complica- 
tions in using anatomic form stable implant through axillari incision.

In terms of implant selection, anatomic form stable implant have definite advantages in the constricted breast, tubular breast, and breasts with tight lower pole for making shape than round silicone gel implant. But the anatomic form stable implants are more firmer, sometimes slight hard in consistency, and unnatural shape when patients are lying down than smooth round silicone gel implant, especially in thin skin and soft tissue patients, common in oriental women. And anatomic form stable implant should not be used when the skeletal deformities of the chest wall, such as concave rib cage, convex rib cage, and irregular rib cage, are evident. So the oriental women should be told about advantages and disadvantages of the anatomic form stable implant before surgery.

\section{CONCLUSION}

Anatomic form stable implant has some benefits such as less ripples, lower pole expansion property, and less conspicu- ous upper pole in thin patients. and IMF incision is the most predictable route for these implants. But if surgeons insert anatomic form stable implants through the axilla, surgeons should consider how to insert gently and maintain steadily. 8 surgeons suggested their own cutting edge method, however each surgeon should find his or her own method. Authors and KAABS hope that these developing and incomplete concepts help beginners to find their own concepts.

\section{REFERENCES}

1. Ahn ST: Breast Augmentation. In Sangtae Ahn. Aesthetic and Reconstructive Breast Surgery. Seoul, Goonja, 2010, p 3

2. Park JS: Primary Breast Augmentation with Anatomical Form-stable Implant. Arch Aesthetic Plast Surg 19: 7, 2013

3. Hammond DC: Atlas of aesthetic breast surgery. Elsevier Inc. 2009, p 19

4. Randquist $\mathrm{C}$ and Gribbe O: Form stable shaped high cohesive gel implants. In Hall-Findlay EJ and Evans GR: Aesthetic and Reconstructive Surgery of the Breast. Elsevier Inc, 2010, p 339

5. Sim HB, Wie HG, Hong YG: Endoscopic transaxillary dual plane breast augmentation. J Korean Soc Plast Reconstr Surg 35(5): 546, 2008 